\title{
A new user-based system for historical city centres
}

\author{
E. M. Cepolina, M. Bonfanti \& A. Farina \\ Department of Civil and Industrial Engineering, University of Pisa, Italy
}

\begin{abstract}
The paper concerns the conceptual design of a new car-sharing system for historical city centres and the simulation of the system for the historical city centre of Genoa. The application fields of the system are historical city centres characterised by narrow streets and interactions with high pedestrian flows. The proposed transport system is based on a fleet of Personal Intelligent City Accessible Vehicles (PICAV). The following specific services are provided: instant access, open ended reservation and one way trips. Vehicles can be accessed and returned only at parking lots. These parking lots are placed in locations on the historical city centre border close to interchange points with public transportation. PICAV users are generated at these parking lots during the simulation. We assume the existence of a system supervisor who is in charge of addressing at least part of the PICAV users to specific parking lots. The supervisor, according with: the current waiting times at parking lots; the number of PICAV units available in each parking lot; the choice set $\mathbf{D}_{i}$ of user $i$; assigns a parking lot to the user that has to be reasonable from both the point of view of the flexible user and of the fleet manager. An object-oriented simulator has been developed. Given an area, the localization of the PICAV parking lots, the fleet of PICAV units at each parking lot at the beginning of the simulation time and the PICAV transport demand, the simulator is able to provide the distribution of waiting times at each parking lot. This result enables us to dimension the fleet in order to have a given percentile of the distribution of user waiting times less than a given threshold.
\end{abstract}

Keywords: car sharing transport system, user based relocation, object-oriented micro simulation. 


\section{Introduction}

A new car-sharing transport system is here proposed. It aims at providing an efficient and rational service to citizens in urban areas where private traffic is restricted because of high pedestrian flows and environmental issues (pedestrian areas) and conventional public transport services cannot operate because of the width and the slope of infrastructures (historical city centers).

The new transport system is based on the use, in car-sharing mode, of a fleet of personal intelligent vehicles (PICAV) [1]. These vehicles, because of their small dimensions, on-board intelligence and zero environmental impact (they are electrically powered), are suitable for moving in these areas disturbing the pedestrian flows as little as possible. These vehicles will be very small, about $1.1 \mathrm{~m}$ wide and $1.3 \mathrm{~m}$ long, and they are equipped with some sensors, which allow them to move without the need of a driver [2]. The proposed transport system integrates with public transport, in areas where neither buses nor cars can operate, and acts as a small link between walking, bicycle and conventional public transport. It is meant to ensure accessibility to everybody, especially elderly and disabled people. It is an on-demand system. It tries to overcome the barriers of traditional car-sharing system, providing these specific services: open ended reservation, instant access and one-way trips.

The PICAV vehicles are available in several parking lots placed all around the pedestrian area, close to interchange points with public transport. The users of this transport system reach the pedestrian area through public transport, take the PICAV vehicle, perform all their activities, then return the vehicle in a parking lot and then take the public transport again to go back home.

Open ended reservation, instant access and one-way trips make the proposed transport system highly flexible and innovative. However, this leads to the critical problem of an imbalance of vehicles distributed among parking lots. Several systems resolve imbalance problems through operator based strategies [3], but these incur high staff and management costs. In order to solve this problem or at least to reduce it, we propose a fully user based relocation: in this scheme, admit the existence of a system supervisor who is in charge of choosing the parking lot where some users, called flexible users, have to return the vehicle.

A simulator of the proposed transport system has been developed. It is able to reproduce the second-by-second activity of each user and of each PICAV unit and therefore results in a useful tool for analyzing "what-if” scenarios.

The transport system has been planned for the historical city center of Genoa.

The paper is structured in this way: section 2 describes the proposed transport system and section 3, its modeling. Section 4 concerns the case of study, and section 5 , the simulation of the proposed transport system. Conclusions follow.

\section{The proposed transport system}

We make reference to a time period starting at $6 \mathrm{am}$ and ending at 0:00pm and we assume to be constant the demand for the PICAV transport system in a sequence of time periods. We take into account only visits to the pedestrian area in the 
reference time period: i.e. people live outside the given pedestrian area, reach the pedestrian area border by conventional transport, pick up a PICAV unit in a parking lot, perform a trip within the urban area by PICAV, return the PICAV unit in a parking lot and go back home again by public transport.

The users may perform one or more activities during their trip within the pedestrian area: if the activity takes longer than one hour, the PICAV unit has to be returned in a parking lot, otherwise the user keeps the PICAV unit and parks it for a short time $(<1 \mathrm{~h})$ along the street, close to his activity place.

The types of trips performed by PICAV users can be classified as follows.

Type A: Multi Mission trip. A multi mission is a trip characterized a series of stops (whose duration depends on the time required to perform an activity which can last from few minutes to dozens of minutes, but never more than one hour), and short trips on board the PICAV unit between successive stops. The trip chain can be circular or linear, i.e. the PICAV user may, at the end of his journey, return the vehicle to the same parking lot of origin or to a different one. This type of trip has both origin and destination at parking lots on the border of the intervention area.

Type B: Single Mission trip. This trip is a linear displacement origin destination without any intermediate stop. The origin is a parking lot located on the border of the intervention area; the destination is the parking lot closer to the location where the PICAV user has to perform a long term activity (whose duration is greater than one hour). The destination parking lot is inside the intervention area.

Type C: Return trip. This trip is the return of a type B trip. Therefore the origin parking lot is the destination parking lot of a type $\mathrm{B}$ trip. The destination parking lot of a type $\mathrm{C}$ trip is on the border of the area, close to a stop from which the user can suitably reach home by public transport.

\section{The transport system modeling}

The main components of the proposed transport system are: the network, the PICAV user, the PICAV unit and the system supervisor.

\subsection{The network}

The network is composed of parking lots and nodes.

\subsubsection{Parking lots}

The parking lot is a station where users pick up and return PICAV units. Therefore, parking lots are the origins and destinations of all the trips by PICAV units. PICAV units recharge whilst idle at parking lots. Parking lots could be located on the border of the pedestrian area or inside it, in the proximity of:

- interchange points, such as: public transport stops, parking lots reserved for disabled people, parking lots for private vehicles;

- attractors where activities that require stops longer than one hour take place: e.g. hotels, museums, offices, schools and universities, etc. 
Each parking lot $P L_{j}$ is characterized by:

- the number of available PICAV units at the beginning of the time period;

- the attractivity: the probability that a single mission trip ends in a given parking lot depends on the parking lot's attractivity. It is a function of number and typology of attractors within the parking lot's influence area;

- a vector of waiting times $t w_{P L j}(t)$ : its dimension equals the number of users currently waiting for a PICAV unit at the given parking lot and each component is the user's current waiting time.

\subsubsection{Nodes}

The node is a point, in the pedestrian area, where a PICAV user can stop for a short time (less than one hour) to do some shopping or activity. The streets inside the pedestrian area have been divided into units and at the center of gravity of each unit a node is located. We assign an attractivity to each node, which is a function of the number and the typology of the attractors presents in the unit. We considered the following attractors: grocery stores, bars and restaurants, pharmacies, clothing stores, furniture stores, craft shops and antiques, varied. The probability that a PICAV user stops at a given node for a short term activity depends on the node's attractivity.

\subsection{PICAV units}

Each PICAV vehicle has the following attributes:

- the initial condition: the parking lot where it is idle at the beginning of the reference time period;

- $\quad$ the battery capacity and the battery charging and discharging law.

\subsection{PICAV users}

Each PICAV user $i$ is described by the following attributes:

- the initial condition $C_{i 0}=\left(t_{i 0}, L_{i 0}\right)$ where:

- $t_{i 0}$ is the time instant in which the user $i$ arrives at a parking lot;

- $L_{i 0}$ is the parking lot in which the user $i$ arrives;

- the activity - travel pattern: $\left(T_{i}, L_{i}\right)=\left(T_{i 1}, \ldots, T_{i n} ; L_{i 1}, \ldots, L_{i n}\right)$; where:

- $\quad T_{i j}$ is the duration of the $j^{\text {th }}$ activity performed by user $i$ in the area;

- $\quad n$ is the number of activities carried out by the user $i$;

- $\quad L_{i j}$ is the node in which the user i performs the $j^{\text {th }}$ activity;

- $\quad$ the user's choice set $\mathrm{Di}=\left(P L_{1}, \ldots, P L_{j}, \ldots, P L_{b}\right)$; where $P L_{j}$ is a parking lot which is convenient for the user $i$. In fact, close to it, the user can catch a bus to go back home. Users whose choice set embraces more than one parking lot, are flexible users.

\subsection{The system supervisor}

When a flexible user is going to complete his activity within the pedestrian area (type A or type $\mathrm{C}$ missions), he calls the system supervisor to ask him where to 
return the PICAV unit. The supervisor assigns to the flexible user a parking lot $D_{i, F}$ within the user's choice set $D_{i}$. The supervisor's choice is a function of:

- $\quad$ the choice set of user $i: \mathbf{D} i=\left(P L_{1}, \ldots, P L_{j}, \ldots, P L_{b}\right)$;

- the current maximum waiting time $t w_{P L j}^{\max }$ at each parking lot $P L_{j}$ :

$$
\forall P L_{j} \in \mathbf{D}_{i}: \rightarrow\left(t w_{P L_{1}}^{\max }, \ldots, t w_{P L_{j}}^{\max }, \ldots, t w_{P L_{b}}^{\max }\right)
$$

- the estimated travel time between the user $i$ 's current position and the parking lot $P L_{j}: t t_{P L j}^{i}$ :

$$
\forall P L_{j} \in \mathbf{D}_{i}: \rightarrow\left(t t_{P L_{1}}^{i}, \ldots, t t_{P L_{j}}^{i}, \ldots, t t_{P L_{b}}^{i}\right)
$$

The system supervisor addresses the flexible user $i$ to the parking lot $\mathbf{D}_{i, \mathrm{~F}}$ for which it results:

$$
\frac{t w_{\mathbf{D}_{F}}^{\max }}{t t_{\mathbf{D i}_{F}}^{i}}=\max _{\forall P L_{j} \in \mathbf{D}_{i}}\left(\frac{t w_{P L_{j}}^{\max }}{t t_{P L_{j}}^{i}}\right)
$$

\section{Application to the historical city center of Genoa}

\subsection{Definition of the intervention area}

The intervention area is the historical city center of Genoa. It is a pedestrian area where public transport cannot operate because of the narrowness of streets and where private vehicles are not allowed to circulate. The historical city center is well served by public transport (buses and underground), whose lines serve the overall border of the historical area $[4,5]$. Citizens are discouraged to reach the city centre border by private modes since in the surrounding area, zone parking is charged [6].

\subsection{Interviews}

Two days of interviews have been performed in order to study the characteristics of trips performed within the historical city center, according to the methodologies for transport demand estimation proposed in [7]. The first day of interviews was carried out on December 3 in the time interval between 4 and 5.30pm; the second one was performed on March 18 between 11.30am and $1.30 \mathrm{pm}$ and between $4.30 \mathrm{pm}$ and $6.15 \mathrm{pm}$. We interviewed people waiting for the bus or train at five bus stops and three underground parking lots close to the historical city center border (see figure 1).

Firstly, we asked each person if they were exiting the historical city center, then in the case of an affirmative answer to this question, we asked for a description of the activity travel patterns carried out in the historical city center. The activity travel pattern description concerns the sequence of activities carried out, the time spent for each activity, the typology of the activity and the path between two successive activities. The number of the interviewed people is equal to 365 . The results show that about $45.5 \%$ (166/365) of the interviewed people 
were exiting the historical city center and among them 39\% (65/166) work or study in the historical city center. A summary of collected data is shown in figure 2.
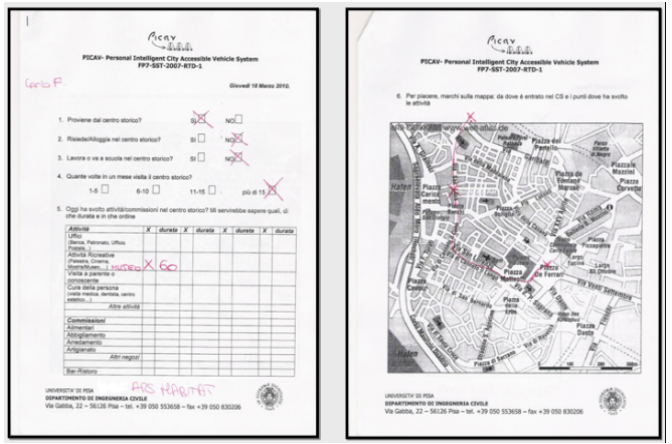

Figure 1: $\quad$ A sample questionnaire.

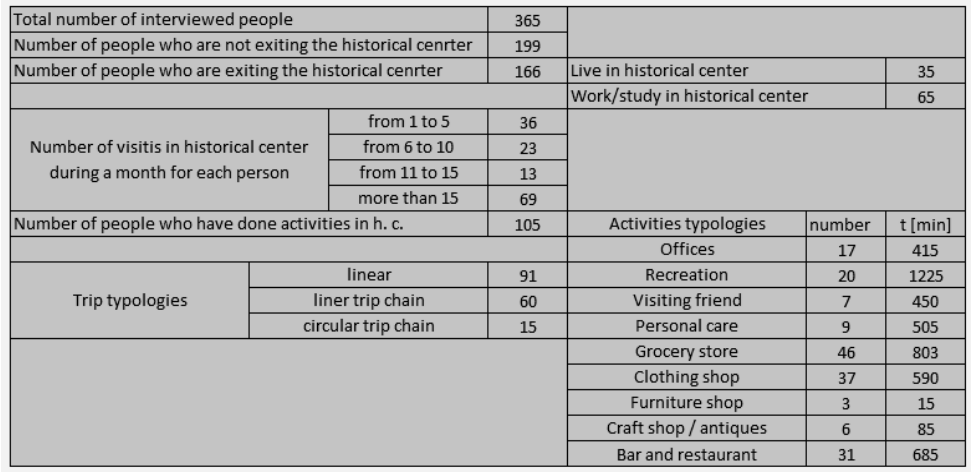

Figure 2: $\quad$ The summary of the collected data.

Among the 166 people coming from the historical city center, we have:

- 55\% (91/166) have performed a trip without intermediate stops;

- 36\% (60/166) have performed a linear trip chain;

- $9 \%(15 / 166)$ have performed a circular trip chain.

\subsection{The network}

\subsubsection{The disposition of parking lots}

We designed nine parking lots, among which seven are located on the border of the intervention area, indicated by: $P L_{1}, P L_{2}, P L_{3}, P L_{4}, P L_{5}, P L_{6}$ and $P L_{7}$, at the most important (in terms of passenger flows) stops; and only 2 are located within the historical city center, near to areas of artistic and/or socio-cultural interest, and they are indicated by: $P L_{10}$ and $P L_{11}$. 


\subsubsection{Attractivity of parking lots}

We determined the attractivity of the nine parking lots. We have identified for each parking lot its area of influence, which we assumed of circular shape and a radius of $300 \mathrm{~m}$. Within each area we have identified the type and the number of activities that require more than one hour stop. The attractivity of each parking lot has been assessed as a function of the number and typology of the activities within its influence area [8]. The probability that a PICAV user, who performs a type B trip, ends it in a given parking lot is proportional to the parking lot's attractivity. The localization of parking lots as well as their attractivity are shown in figure 3.

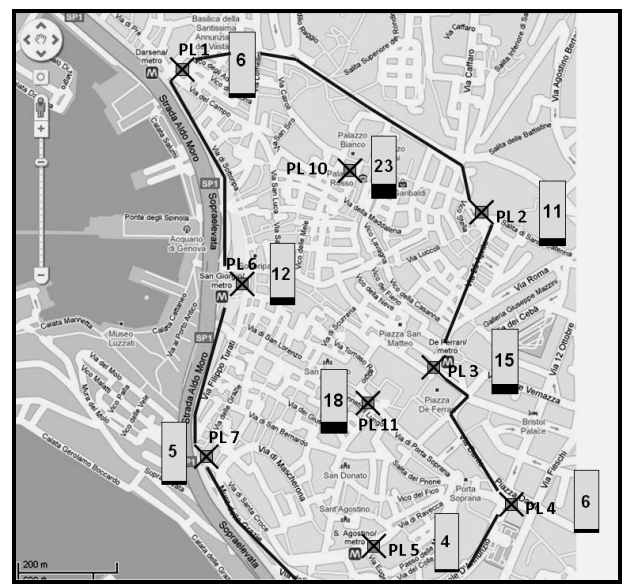

Figure 3: The attractivity of each parking lot.

\subsubsection{Attractivity of nodes}

We calculated the attractivity of each node. We divided the streets of the historical city center in 120 units whose length is $50 \mathrm{~m}$. In each unit we have identified the type and number of commercial activities that require a short term stop (from few minutes to one hour). Therefore, on the basis of the number and typology of these activities within each unit, we have assigned to each node (center of gravity of each unit) a probability that a type A user calls the supervisor from the node. The position of nodes and the distribution of attraction in the area are displayed in figure 4.

\subsection{Characterization of the PICAV transport demand}

\subsubsection{Temporal distribution of PICAV transport demand}

We determined the temporal distribution of the PICAV users during the simulation time. We obtained, from the data provided by AMT (the municipal public transport company in Genoa) [4, 5], for each public transport stop on the historical city center border, the number of arrivals from 6am to 9am, i.e. the number of passengers that got off the public transport and did not get on new 


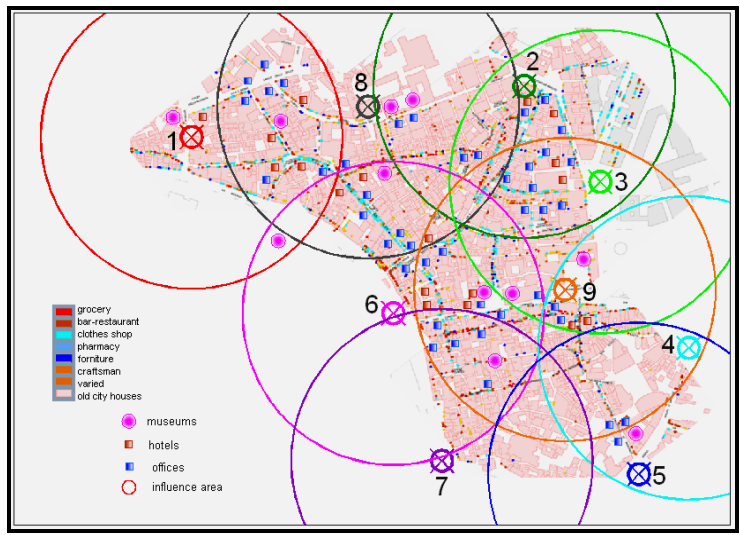

Figure 4: Distribution of attractors in the historical city centre of Genoa.

lines. We extrapolated the known number of arrivals at the bus stops on the border (from 6am to 9am.) to the whole simulation time period. In order to determine the temporal distribution of the users during the simulation time, several videos have been recorded of pedestrian densities in different time periods, on the study area. The results have shown that the afternoon demand is about 1.45 times the morning demand, and that the afternoon period begins at about 4pm. According to the results of the video, to data taken from the AMT website and to direct observations, two periods of demand have been assumed: an off-peak period, which ranges from $8 \mathrm{am}$ to $4 \mathrm{pm}$; a peak period, from $4 \mathrm{pm}$ to 8pm. From 8pm to midnight there is no PICAV transport demand.

From the results of the interviews, we know that, among the total number of passengers who arrive on the border of the historical city center, only $45 \%$ actually enter into the intervention area. We assumed that, among the total number of travelers who enter the intervention area, only $1 \%$ might actually use the PICAV unit.

\subsubsection{Spatial distribution of PICAV transport demand}

We assessed the probability that a PICAV user arrives at each parking lot on the border of the historical city center, in this way:

- We identified for each parking lot its area of influence; we assumed circular shape and a radius of $300 \mathrm{~m}$.

- We identified the public transport stops within each area.

- For each parking lot we summed the number of arrivals from 6am to 9am at all the stops within the parking lot's influence area.

- The probability to have an arrival at a given parking lot is assessed as the rate between: the number of arrivals assessed in the previous step, and the overall number of arrivals at the historical city center border in the same time period.

The spatial distribution of arrivals to parking lots is shown in figure 5 . 


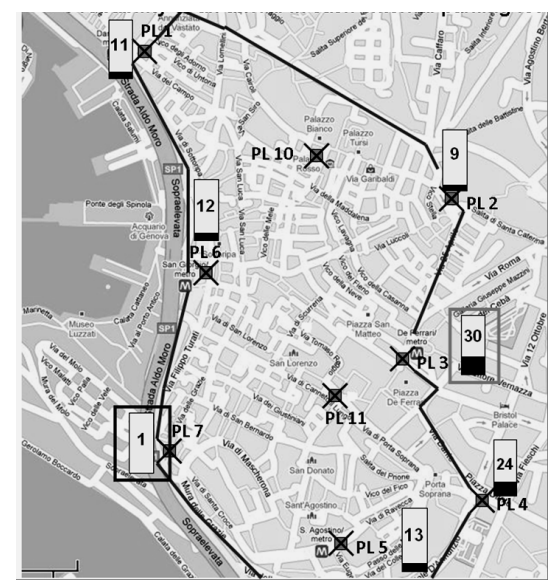

Figure 5: The spatial distribution of arrivals at parking lots on the border.

\subsubsection{Trip typologies}

From the collected data, we obtained the following information about the typologies of trips performed in the historical city centre.

- $\quad 45 \%$ are type A trips; the average number of activities in the activity travel pattern is 1.7; the average duration of the activity chain is 40 minutes.

- $55 \%$ of PICAV users perform a type B trip (PICAV users who perform a type $\mathrm{C}$ trip are the same number); the average trip duration is 2.5 minutes.

\subsubsection{Choice set of flexible users}

We divided the metropolitan area of Genoa into three macro areas: North, East and West [8], and for each we determined:

- the percentage of residents;

- the number of lines of public transport which serve each macro area.

The first piece of information is used to assign a macro area to each PICAV user.

The second piece of information is used to assign a choice set to each macro area. This choice set is composed by all the stops where a public transport line, suitable to reach the macro area, stop. The related choice sets are shown in figure 6 .

\section{Simulation}

A simulator of the proposed transport system has been developed. The code has been written with Python 2.6 language [9], which is a free piece of software and uses an object-oriented approach. The input data are: the location of nodes, the location of parking lots, fleet distribution among parking lots at the simulation starting time, the demand for PICAV units and its temporal and spatial distribution. The output data is the distribution of users' waiting times in each parking lot. 


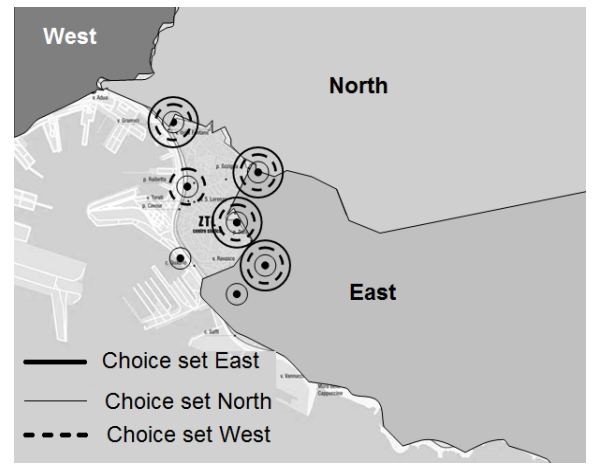

Figure 6: The attractivity of each macro area.

\subsection{Scenarios}

Using the simulator, we have analyzed three scenarios to see how the transport system performs. The three scenarios differ only in the total number of PICAV units that form the fleet and thus the number of units in each parking lot at the beginning of the simulation time. A uniform distribution of vehicles among parking lots at the beginning of the simulation time has provided higher waiting times than a nonuniform distribution of vehicles. Several runs of the simulator have been performed in order to determine a distribution of the fleet which keeps the waiting times low enough. The output of the program is the distribution of the users' waiting times, from which we have calculated the average and the $50^{\text {th }}$, $90^{\text {th }}$ and $95^{\text {th }}$ percentiles. These values are often different from a run of the programme to the other, therefore, in order to eliminate the stochastic effect caused by the many random quantities presents in the simulator, 30 runs have been performed for each scenario. In order to evaluate the transport system's Level of Service (LOS) for what regards the users' waiting times, Castangia and Guala [10] provided the criteria shown in table 1, according to the values of the $50^{\text {th }}, 90^{\text {th }}$ and $95^{\text {th }}$ percentiles of users' waiting times.

Table 1: LOS definition based on the statistical distribution of waiting times [10].

\begin{tabular}{|c|c|c|c|}
\hline \multirow{2}{*}{ LOS } & \multicolumn{3}{|c|}{ Waiting time (minutes) not greater than: } \\
\cline { 2 - 4 } & $50^{\text {th }}$ percentile & $90^{\text {th }}$ percentile & $95^{\text {th }}$ percentile \\
\hline A & 0.5 & 1 & 1.5 \\
\hline B & 1 & 2 & 3 \\
\hline C & 1.5 & 3 & 5 \\
\hline D & 2.5 & 5 & 8 \\
\hline E & 4 & 8 & 10 \\
\hline F & worse & worse & worse \\
\hline
\end{tabular}




\subsubsection{Scenario 1}

The first scenario assumes a fleet of 73 PICAV units, and their distribution among parking lots is the following: $(8,9,10,10,11,7,6,6,6)$.

The simulation output shows that the average wait time is equal to 2.75 minutes, the $95^{\text {th }}$ percentile is equal to 10.7 minutes, the $90^{\text {th }}$ percentile is equal to 4 minutes and the $50^{\text {th }}$ percentile is equal to 0.9 minutes. According to table 1 , the LOS of the transport system is between $\mathrm{E}$ and $\mathrm{F}$.

\subsubsection{Scenario 2}

The second scenario assumes a fleet of 82 PICAV units, i.e. 1 vehicle for each parking lot more than the previous scenario. The distribution of vehicles therefore has been assumed the same as before. In this scenario, the $95^{\text {th }}$ percentile of users' waiting times is equal to 3.4 minutes, the $90^{\text {th }}$ percentile is equal to 1.1 , and the $95^{\text {th }}$ percentile is 0 . The average waiting time is equal to 0.85 minutes. This scenario is much better and it provides a level of service $\mathrm{C}$.

\subsubsection{Scenario 3}

The last scenario assumes a fleet of 91 PICAV units, i.e. 1 vehicle for each parking lot more than the previous scenario. The simulation output shows that the average waiting time is equal to 0.25 minutes, the $95^{\text {th }}$ percentile is equal to 0.45 minutes and the $90^{\text {th }}$ percentile is equal to 0.19 minutes. This scenario is definitely the best from the point of view of user, because it drastically reduces waiting times. From the perspective of the operator, instead, it has the problem of a wide number of PICAV units, resulting in high cost of acquisition and fleet management.

\subsection{Simulation results}

The results for the three scenarios exposed above are shown in figure 7. Also the scenarios of a fleet dimension of 100 and 19 vehicles have been investigated. The results show that increasing the fleet dimension, from 73 to 82 vehicles, we have a high reduction in users' waiting times, especially with reference to the

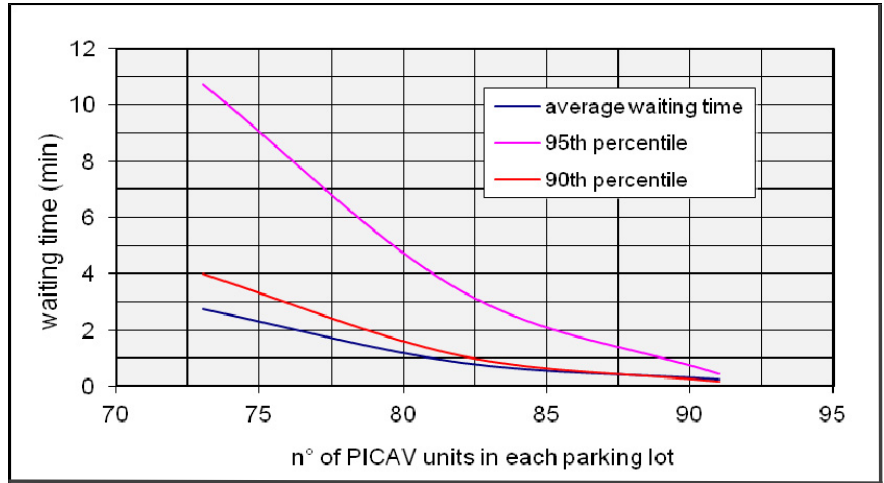

Figure 7: The simulation's results. 
$90^{\text {th }}$ and $95^{\text {th }}$ percentiles. Further increasing the fleet dimension, we still have a reduction of waiting times, but it is really minor, especially if compared with the much higher costs for the acquisition and management of the fleet.

\section{Conclusions}

The paper concerns a new car-sharing system, with a low environmental impact, for pedestrian areas, which is meant to provide to citizens an efficient and rational service in areas where private traffic is restricted and conventional public transport cannot operate. This transport system is part of the European project PICAV, Seventh Framework Program Theme [SST - 212 - RTD - 1].

In section 4.3, three scenarios have been investigated. Among them, the best scenario, in order to compensate both the user and the system's points of view, is the situation of 82 vehicles of fleet dimension. A nonuniform initial distribution of vehicles among parking lots has been hypothesized, in order to keep lower the distribution of users' waiting times. This provides better results than a uniform distribution of vehicles, but this distribution is simply a result of some simulation runs. A lower fleet dimension than 82 vehicles or a higher level of service of the system can be achieved under a better distribution of vehicles at the beginning of the simulation period. In [11], an optimization procedure of the proposed transport system has been developed, which is aimed to determine the best fleet dimension and distribution among stations, in order to minimize the total waiting time of PICAV users as well as the transport system's cost.

The proposed transport system has been developed under the hypothesis of the flexibility of users. However, this hypothesis works properly in a scenario like Genoa, in which some parking lots are indifferent to users returning PICAV vehicles. Several other scenarios exist in which this hypothesis cannot be made, in which, for example, users reach the study area by private car, or by train, therefore they do not have a choice set or their choice set is limited to 1 or 2 parking lots. As stated in the introduction, operator based strategies incur high staff and management costs. Therefore, future work can be the study of relocation procedures, which have lower staff costs than operator-based strategies, and can be applied also in scenarios where the hypothesis of users' flexibility cannot be made.

\section{References}

[1] http://www.dimec.unige.it/pmar/picav/

[2] Masood, J., Zoppi, M., Molfino, R., Multi-Terrain Vehicle Active Suspension Control Design and Synthesis. $7^{\text {th }}$ International Conference on Mechatronics and Embedded Systems and Applications, Washington DC, August, 2011.

[3] Cepolina, E.M., Farina, A. Urban car sharing: An overview of relocation strategies. In: Longhurst, J.W.S., Brebbia, C.A. (Eds.), Urban Transport XVIII. WIT Press, Southampton, UK, pp. 419-431, 2012.

[4] http://www.amt.genova.it 
[5] http://www.metrogenova.com

[6] http://www.bluareagenova.it

[7] Ferrari, P., Fondamenti di Pianificazione dei Trasporti, Pitagora Editrice, Bologna, 2001.

[8] http://www.comune.genova.it

[9] http://www.python.it

[10] Castangia, M., Guala, L., Modelling and simulation of PRT networks. In: Pratelli, A., Brebbia, C.A. (Eds.), Urban Transport XVII. WIT Press, Southampton, UK, pp. 459-472, 2011.

[11] Cepolina, E.M., Farina, A., A new shared vehicle system for urban areas. Transportation Research C, Vol. 21, Issue 1, pp. 230-243, 2012. 